\title{
The heat semigroup and equation related to a Bessel-type operators and the canonical Fourier Bessel transform
}

\author{
Ghazouani Sami ${ }^{1}$ and Sahbani Jihed ${ }^{2}$ \\ ${ }^{1}$ University of Carthage, Faculty of Science of Bizerte, (UR17ES21),"Dynamical systems \\ and their applications" 7021, Jarzouna, Bizerte,Tunisia \\ ${ }^{2}$ Faculty of Sciences of Tunis, University of Tunis El Manar, 2092 Tunis, Tunisia
}

June 8, 2021

\begin{abstract}
In this paper we study a translation operator associated with the canonical Fourier Bessel transform $\$ \backslash \operatorname{mathcal}\{\mathrm{F}\}_{-}\{\backslash \mathrm{nu}\}^{\wedge}\{\backslash \mathrm{mathbf}\{\mathrm{m}\}\} \$$ We then use it to derive a convolution product and study some of its important properties. As a direct application, we introduce the heat semigroup generated by the Bessel-type operators $\$ \$ \backslash \operatorname{Delta}_{-}\{\backslash \mathrm{nu}\}^{\wedge}\left\{\backslash \operatorname{mathbf}\{\mathrm{m}\}^{\wedge}\{-1\}\right\}=\backslash \operatorname{frac}\left\{\mathrm{d}^{\wedge}\{2\}\right\}\left\{\mathrm{dx}^{\wedge}\{2\}\right\}+\backslash \operatorname{left}($ $\backslash \operatorname{frac}\{2 \backslash \mathrm{nu}+1\}\{\mathrm{x}\}+2 \mathrm{i} \backslash \operatorname{frac}\{\mathrm{a}\}\{\mathrm{b}\} \mathrm{x} \backslash \operatorname{right}) \backslash \operatorname{frac}\{\mathrm{d}\}\{\mathrm{dx}\}-\backslash \operatorname{left}\left(\backslash \operatorname{frac}\left\{\mathrm{a}^{\wedge}\{2\}\right\}\left\{\mathrm{b}^{\wedge}\{2\}\right\} \mathrm{x}^{\wedge}\{2\}-2 \mathrm{i} \backslash \operatorname{left}(\backslash \mathrm{nu}+1 \backslash\right.$ right $) \backslash$ frac $\left.\{\mathrm{a}\}\{\mathrm{b}\} \backslash \operatorname{right}\right)$ $\$ \$$ and use it to solve the initial value problem for the heat equation governed by $\$ \backslash$ Delta $_{-}\{\backslash n u\}^{\wedge}\left\{\backslash \operatorname{mathbf}\{\mathrm{m}\}^{\wedge}\{-1\}\right\} . \$$
\end{abstract}

\section{Hosted file}

The heat semigroup.pdf available at https://authorea.com/users/418649/articles/525327the-heat-semigroup-and-equation-related-to-a-bessel-type-operators-and-the-canonicalfourier-bessel-transform

\section{Hosted file}

The heat semigroup.tex available at https://authorea.com/users/418649/articles/525327the-heat-semigroup-and-equation-related-to-a-bessel-type-operators-and-the-canonicalfourier-bessel-transform 